\title{
Activation of calcineurin and smooth muscle myosin light chain kinase by Met-to-Leu mutants of calmodulin
}

\author{
Robert A. EDWARDS ${ }^{\star}$, Michael P. WALSH†, Cindy SUTHERLAND $†$ and Hans J. VOGEL*1 \\ *Department of Biological Sciences, University of Calgary, Alberta, Canada T2N 1N4, and †Department of Medical Biochemistry, University of Calgary, Calgary, Alberta, \\ Canada T2N 4N1
}

\begin{abstract}
The effects of replacement of each of the individual Met in calmodulin $(\mathrm{CaM})$ with Leu on the activation of two CaM target enzymes [smooth muscle myosin light chain kinase (smMLCK) and calcineurin $(\mathrm{CN})]$ were investigated. The $K_{\mathrm{D}}$ and $P_{\max }$ (percentage maximal activation) values for activation of both enzymes by M76L-CaM were indistinguishable from wild-type (wt)-CaM, which is consistent with the location of Met-76 in the central linker that is not involved in target protein interaction. The other eight Met in CaM are exposed in the hydrophobic surfaces that are involved in target-enzymes binding, and in general equivalent effects are observed for substitutions of Leu for Met residues in homologous positions in the two CaM domains. However, the importance of the interaction of specific Met residues with the target enzyme depends on the particular enzyme. Leu substitution at Met-36 or Met-109 reduced the affinity of MLCK for the mutant and the maximal activation of CN. MLCK had a higher $K_{\mathrm{D}}$ for M51L-CaM whereas M124L-
\end{abstract}

CaM activated the kinase to only $68 \%$ of maximal activity induced by wt-CaM; these mutants were indistinguishable from wt-CaM in activation of CN. M71L- and M144L-CaMs behaved like wt-CaM in activation of MLCK, but activated the phosphatase to only about $80 \%$ of maximal activity induced by wt-CAM. M72L-CaM exhibited an increased affinity for MLCK compared to wt-CaM and slightly decreased maximal activation, whereas M145L-CaM exhibited maximal activation significantly greater than that due to wt-CaM; these mutants behaved like wt$\mathrm{CaM}$ with respect to $\mathrm{CN}$ activation. Finally, a mutant $\mathrm{CaM}$ in which all four C-terminal Met were replaced by Leu $\left(\mathrm{M}_{4}-\mathrm{CT}-\mathrm{L}_{4}-\right.$ $\mathrm{CaM}$ ) had similar affinities for MLCK and CN as wt-CaM but maximal activation of these enzymes by this mutant was only $60-70 \%$ of that achieved with wt-CaM. These results imply that, in addition to removing the autoinhibitory domain from the active site of the target enzyme, CaM must induce a conformational change in the active site itself.

\section{INTRODUCTION}

Calmodulin $(\mathrm{CaM})$ mediates the regulatory effects of $\mathrm{Ca}^{2+}$ on diverse physiological processes such as smooth muscle contraction and neurotransmitter release [1-3]. CaM is composed of two globular domains (each capable of binding two $\mathrm{Ca}^{2+}$ ions) that are connected by a long central $\alpha$-helix in the crystal structure [4]. $\mathrm{Ca}^{2+}$ binding to $\mathrm{CaM}$ induces a conformational change which exposes two Met-rich hydrophobic patches, one in each domain, that are involved in target protein interaction and activation. Some 30 or more CaM-binding proteins have been identified to date. CaM-binding domain sequences show little similarity in their amino acid sequence but the vast majority of them share the propensity to form a basic amphiphilic $\alpha$-helical structure composed of two bulky hydrophobic 'anchor' residues separated by 12 amino acids $[5,6]$. The Met residues of $\mathrm{CaM}$ are highly conserved and are thought to account for its ability to bind to such a large number of target proteins $[3,7,8]$.

Previous studies have shown that mutation of individual methionines that form part of the target-enzyme binding site of CaM significantly affects the activation of some target enzymes while having no effect on others [8-11]. We have extended these studies to an analysis of the effects of mutation in which individual methionines in CaM have been replaced by Leu on activation of smooth muscle myosin light chain kinase (smMLCK) and type $2 \mathrm{~B}$ protein serine/threonine phosphatase (calcineurin, $\mathrm{CN}$ ). The objective of this study was to make minimal changes to the structures of the Met side chains and to evaluate their importance in binding to and activation of two target enzymes with quite distinct affinities for CaM. Mutation of Met to Leu provides slightly increased hydrophobicity of the side chain [12,13], alters its shape [6] and reduces its flexibility and polarizability [7]. CaM contains nine Met residues: four in the N-terminal domain, one in the connecting linker (Met-76) and four in the C-terminal domain in positions homologous to those in the N-terminal domain $[4,5,14]$. Ten mutant CaMs were examined: in nine, the methionines were individually replaced by Leu and, in the tenth, all four C-terminal methionines were replaced by leucines.

\section{MATERIALS AND METHODS}

Wild-type and mutant CaMs were overexpressed and purified from E. coli cells as described previously [8]. All mutants exhibited $\mathrm{Ca}^{2+}$-dependent exposure of a hydrophobic surface since they interacted with phenyl-Sepharose in a $\mathrm{Ca}^{2+}$ dependent manner [8]. CaM concentrations were determined using the $A_{1 \mathrm{~cm} .276}^{1 \%}$ value of 1.8 determined by Watterson et al. [15] and a molecular mass of $16.7 \mathrm{kDa}$ which was calculated from the amino acid composition. The synthetic peptide substrate of smMLCK, KKRPQRATSNVFA, corresponding to residues $11-23$ of the $20 \mathrm{kDa}$ light chain of smooth muscle myosin, was synthesized in the University of Calgary Peptide Synthesis Core Facility using a Beckman model 990B automated peptide synthesizer and purified by preparative reverse-phase HPLC as described previously [16]. The peptide was shown to be $>95 \%$ pure by analytical HPLC

Abbreviations used: CaM, calmodulin; CN, calcineurin; skMLCK, skeletal muscle myosin light chain kinase; smMLCK, smooth muscle myosin light chain kinase; wt, wild-type; $\mathrm{M}_{4}-\mathrm{CT}-\mathrm{L}_{4}$, C-terminal mutant with four Met $\rightarrow$ Leu; PDE, cyclic nucleotide phosphodiesterase; cNOS, constitutive nitric oxide synthetase; nNOS, neuronal nitric oxide synthetase; CaM kinase II, CaM-dependent protein kinase II; CaM kinase IV, CaM-dependent protein kinase IV; $P_{\max }$, percentage maximal activation relative to wt-CaM; RMSD, root mean square difference.

To whom correspondence should be addressed. 
and its structure verified by amino acid composition analysis. $\mathrm{CN}$, purified from bovine brain purified as described previously [17] was generously provided by Dr. Jerry Wang (Hong Kong University of Science and Technology). Concentrations of calcineurin were determined using a $A_{1 \mathrm{~cm} .280}^{1 \%}$ value of 9.26 [18] and a molecular mass of $75 \mathrm{kDa}$ [19]. CN activity was assayed in triplicate in the presence of $1.5 \mathrm{mM} \mathrm{Ca}^{2+}$ with $p$-nitrophenol phosphate as substrate, as described by Newton et al. [20] except that the concentration of $\mathrm{CN}$ was $2 \mathrm{nM}$ and the assay was allowed to proceed for $30 \mathrm{~min}$.

smMLCK was isolated from chicken gizzard as described by Ngai et al. [21]. The activity of smMLCK $(9.3 \mathrm{nM})$ was assayed

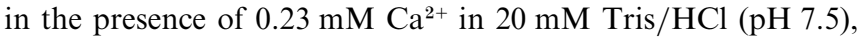
$60 \mathrm{mM} \mathrm{KCl}, 4 \mathrm{mM} \mathrm{MgCl}, 0.2 \mathrm{mM}\left[\gamma^{-32}{ }^{32}\right]$ ATP $(400-500$ c.p.m./ pmol) and the indicated concentration of $\mathrm{CaM}$ or CaM mutants using the peptide substrate at a concentration of $100 \mu \mathrm{M}$. The reaction volume was $30 \mu \mathrm{l}$ and the incubation temperature was $30^{\circ} \mathrm{C}$. Reactions were started by addition of ATP and samples $(20 \mu \mathrm{l})$ were removed after $5 \mathrm{~min}$, spotted onto P81 phosphocellulose paper squares (Whatman) and dropped into $0.5 \%$ $\mathrm{H}_{3} \mathrm{PO}_{4}$. The paper squares were washed three times ( 5 min each) in $0.5 \% \mathrm{H}_{3} \mathrm{PO}_{4}$, rinsed in $\mathrm{CHCl}_{3}$ and dried prior to counting of Cerenkov radiation. Preliminary experiments indicated that substrate phosphorylation was linear for $>5 \mathrm{~min}$. Assays were carried out at least three times, each in triplicate.

The activation data were fitted to a model in which binding of calcium saturated $\mathrm{CaM}(\mathrm{E}+\mathrm{CaM} \rightarrow \mathrm{E} \cdot \mathrm{CaM})$ is required for activation. The enzyme without CaM bound is assumed to be inactive and the $\mathrm{E} \cdot \mathrm{CaM}$ complex has an activity of $100 \%$ for wt$\mathrm{CaM}$ and an activity of $P_{\max }$, which is a variable parameter, for the mutants. The 'best-fit' $P_{\max }$ and $K_{\mathrm{D}}$ values were found by minimizing the root mean square difference (RMSD) between experimental percentage activation (i.e. $P_{\text {exp }}$ ) and the percentage activation calculated (i.e. $P_{\text {calc }}$ ) from trial values of $K_{\mathrm{D}}$ and $P_{\max }$. The fraction of enzyme with $\mathrm{CaM}$ bound (i.e. $R=[\mathrm{E} \cdot \mathrm{CaM}] /$ $\left.[\mathrm{E}]_{\text {Total }}\right)$ is given by the positive roots of the equation $R^{2}+b R+c$ $=0\left\{\right.$ where $c=[\mathrm{CaM}]_{\text {Total }} /[\mathrm{E}]_{\text {Total }}$ and $\left.b=-\left(1+c+K_{\mathrm{D}} /[\mathrm{E}]_{\text {Total }}\right)\right\}$. Thus the quadratic formula allows calculation of $R$ and hence the percentage activity, $P_{\text {calc }}=P_{\max } \times R$. The minimization was done by an evolutionary procedure which made changes in initial trial values of $K_{\mathrm{D}}$ and $P_{\max }$, calculated activation percentages $\left(P_{\text {calc }}\right)$ and saved the result if there was a decrease in the root mean square difference. Data points for which $P_{\exp }<10.0 \%$ were excluded from the fitting procedure, because they have a large relative error and are not crucial for determination of either $K_{\mathrm{D}}$ or $P_{\max }$. The data point for M145L at $5 \mathrm{nM}$ activating smMLCK was an outlier based on a $q$-test so it was also excluded from the fitting procedure.

A $t$ test was used to identify $K_{\mathrm{D}}$ and $P_{\max }$ values for the mutants which are significantly different from the wild type values. The uncertainties in the best-fit $P_{\max }$ values were estimated by using the best-fit $K_{\mathrm{D}}$ value to calculate the $P_{\max }$ predicted by each of the experimental points used in the fitting, for each CaM, then the standard deviations of the sets of $P_{\max }$ values were calculated. A similar procedure was followed to estimate the uncertainties in the best-fit $K_{\mathrm{D}}$ values by using the best-fit $P_{\max }$ to calculate the $K_{\mathrm{D}}$ predicted by each point; then the standard deviation of this set of $K_{\mathrm{D}}$ values was calculated. (The average $P_{\max }$ and $K_{\mathrm{D}}$ values for the sets were not significantly different than the best-fit $P_{\max }$ and $K_{\mathrm{D}}$ values.) Since a multiple comparison is being done (10 mutants are compared to the wild type for each target enzyme), a Bonferroni type of correction was applied in which individual confidence levels of $99.5 \%$ were calculated from $t_{0.995} \sigma / n^{1 / 2}$ (where $n$ is the number of data points) to yield an overall confidence level of $95 \%$ [22].

\section{RESULTS}

The effects of several of the CaM mutants on smMLCK and CN are shown in Figures 1 and 2 and the $K_{\mathrm{D}}$ and $P_{\max }$ values determined from the activation curves are in Tables 1 and 2. In most cases, the activation curves predicted from the simple binding model give reasonable fits to the experimental data. Typically the root mean square difference between the experimental and calculated percentages is $<5 \%$ (Tables 1 and 2 ). However, in the cases of M51L and M145L activation of smMLCK (Figure 1) and the cases of M51L and M124L activation of $\mathrm{CN}$ (Figure 2) the fits are not as good and the root mean square differences are approx. $8 \%$. Observed differences between the mutant and wild type $P_{\max }$ and $K_{\mathrm{D}}$ were not large so it was necessary to estimate the uncertainty using $t$ tests to identify which $K_{\mathrm{D}}$ and $P_{\max }$ values for the mutants were significantly different from the corresponding wt values.

Previous studies have reported that the concentration of $\mathrm{CaM}$ required for half maximal activation of smMLCK is $1-1.3 \mathrm{nM}$ [23-26]. The relatively high value of $K_{\mathrm{D}}$ obtained in this study ( $40.4 \mathrm{nM}$; Table 1) is probably due to different reaction conditions, including use of a synthetic peptide substrate rather than intact myosin or the isolated $20 \mathrm{kDa}$ light chain. $\mathrm{CN}$ is one of the target enzymes which bind CaM very tightly and the $K_{\mathrm{D}}$ of $0.23 \mathrm{nM}$ found for wt-CaM activation of $\mathrm{CN}$ (Table 2) is, within experimental error, the same as the published value of $0.1 \mathrm{nM}$ [27]. Because of such tight binding, the relative uncertainty of the $K_{\mathrm{D}}$ is large so that the decreased affinity of M71L-CaM for CN is the only significant difference from wt-CaM (Table 2).

The $K_{\mathrm{D}}$ and $P_{\max }$ parameters for activation of smMLCK and $\mathrm{CN}$ by M76L-CaM were indistinguishable from those of wt$\mathrm{CaM}$ (Tables 1 and 2). This serves as a useful control because modification in the central linker region, where Met-76 is located,

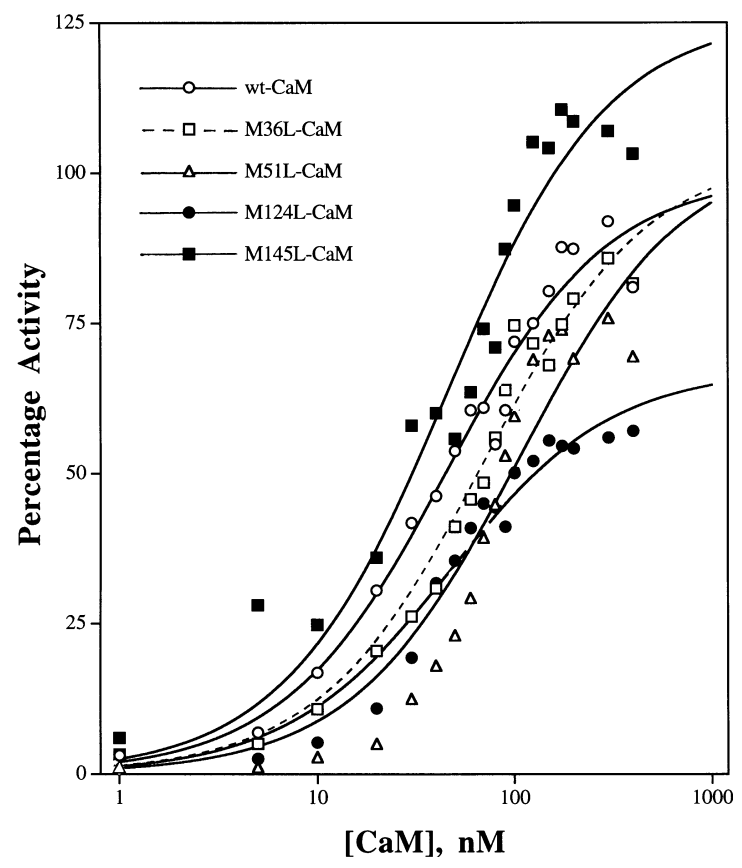

Figure 1 Activation of smMLCK by CaM mutants

Maximal activation by wt-CaM is set at $100 \%$. Data points are indicated by symbols and the lines are drawn from the best-fit $K_{\mathrm{D}}$ and $P_{\max }$ values. Solid lines are drawn for wt-CaM and all mutants which have either a $K_{\mathrm{D}}$ or $P_{\max }$ that is significantly different from the wild type. 


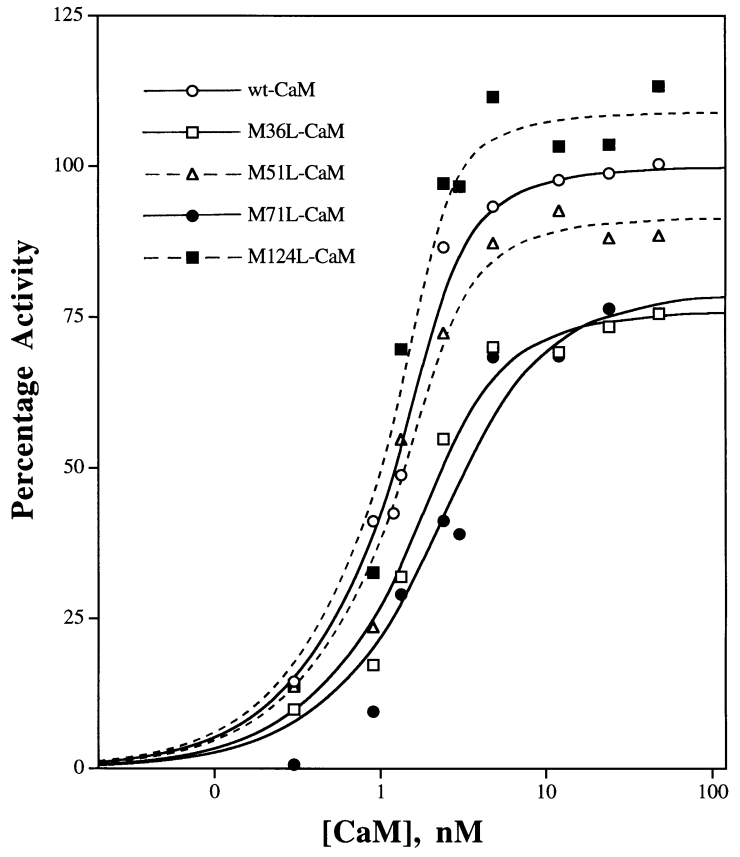

Figure 2 Activation of CN by CaM mutants

Maximal activation by wt-CaM is set at $100 \%$. Data points are indicated by symbols and the lines are drawn from the best-fit $K_{\mathrm{D}}$ and $P_{\max }$ values. Solid lines are drawn for wt-CaM and all mutants which have either a $K_{D}$ or $P_{\max }$ that is significantly different from the wild type.

Table 1 The best-fit parameters for CaM binding and activation of SMMLCK

$K_{D}$ and $P_{\max }$ values are shown with $99.5 \%$ confidence limits. Values which are significantly different from the values for wt-CaM are designated with an asterisk $\left(^{*}\right)$. The RMSDs were calculated from $\left(\Sigma\left(P_{\text {exp }}-P_{\text {calc }}\right)^{2} / \pi\right)^{1 / 2}$.

\begin{tabular}{|c|c|c|c|}
\hline $\mathrm{CaM}$ & $K_{\mathrm{D}}$ in $\mathrm{nM}$ & $P_{\max }$ & RMSD \\
\hline Wild type & $40.4 \pm 13.7(16)$ & $100.0 \pm 5.6(16)$ & 4.7 \\
\hline M36L & $65.2 \pm 13.4(16)^{\star}$ & $103.7 \pm 8.4(16)$ & 4.9 \\
\hline M51L & $100.3 \pm 48.4(14)^{\star}$ & $104.7 \pm 20.6(14)$ & 8.6 \\
\hline M71L & $45.7 \pm 11.9(16)$ & $105.5 \pm 8.5(16)$ & 4.5 \\
\hline M72L & $23.2 \pm 11.9(16)^{\star}$ & $90.8 \pm 6.9(16)^{\star}$ & 5.3 \\
\hline M76L & $52.5 \pm 10.4(16)$ & $102.8 \pm 9.1(16)$ & 3.5 \\
\hline M109L & $70.0 \pm 18.5(15)^{\star}$ & $98.0 \pm 11.6(15)$ & 4.3 \\
\hline M124L & $42.7+16.3(15)$ & $67.5+8.5(5)^{*}$ & 3.8 \\
\hline M144L & $41.7 \pm 7.6(16)$ & $104.8 \pm 6.4(16)$ & 3.9 \\
\hline M145L & $40.9 \pm 14.0(16)$ & $126.6 \pm 10.7(16)^{\star}$ & 7.3 \\
\hline $\mathrm{M}_{4}-\mathrm{CT}-\mathrm{L}_{4}$ & $55.6 \pm 13.7(14)$ & $62.6 \pm 5.5(14)^{\star}$ & 3.3 \\
\hline
\end{tabular}

would be expected to have very little influence on the binding or activation of target enzymes.

Met-36 and Met-109 are in homologous positions in the second helix of each of the two domains. Both M36L- and M109L-CaMs had significantly higher $K_{\mathrm{D}}$ values for smMLCK than wt-CaM, but maximal activation was the same. On the other hand, M36L- and M109L-CaMs had the same $K_{\mathrm{D}}$ values for $\mathrm{CN}$ as wt-CaM; but maximal activation was only $75-80 \%$ of that achieved with wt-CaM (Table 2).

Met-51 and Met-124 are in homologous positions in the third helix of each of the two domains of CaM. M51L-CaM had a significantly higher $K_{\mathrm{D}}$ value for activation of smMLCK than wt-

\section{Table 2 The best-fit parameters for CaM binding and activation of CN}

$K_{\mathrm{D}}$ and $P_{\max }$ values are shown with $99.5 \%$ confidence limits. Values which are significantly different from the values for wt-CaM are designated with an asterisk $\left(^{\star}\right)$. The RMSDs were calculated from $\left(\Sigma\left(P_{\text {exp }}-P_{\text {calc }}\right)^{2} / \eta\right)^{1 / 2}$

\begin{tabular}{|c|c|c|c|}
\hline $\mathrm{CaM}$ & $K_{\mathrm{D}}$ in $\mathrm{nM}$ & $P_{\max }$ & RMSD \\
\hline Wild type & $0.23 \pm 0.14(9)$ & $100.0 \pm 8.9(9)$ & 3.9 \\
\hline M36L & $0.55 \pm 0.75(8)$ & $76.1 \pm 16.2(8)^{\star}$ & 4.4 \\
\hline M51L & $0.26 \pm 0.96(8)$ & $91.6 \pm 21.7(8)$ & 6.1 \\
\hline M71L & $1.2 \pm 1.1(7)^{\star}$ & $79.2 \pm 15.2(7)^{\star}$ & 5.2 \\
\hline M72L & $0.36 \pm 1.4(6)$ & $100.8 \pm 44.1(6)$ & 4.2 \\
\hline M76L & $0.22 \pm 1.7(6)$ & $99.8 \pm 28.3(6)$ & 4.4 \\
\hline M109L & $0.38 \pm 1.3(8)$ & $82.6 \pm 14.4(8)^{*}$ & 5.8 \\
\hline M124L & $0.13 \pm 1.1(9)$ & $109.1 \pm 21.8(9)$ & 7.6 \\
\hline M144L & $0.17 \pm 1.2(8)$ & $81.7 \pm 13.6(8)^{\star}$ & 4.1 \\
\hline M145L & $0.15 \pm 0.35$ & $94.6 \pm 23.2(5)$ & 3.4 \\
\hline $\mathrm{M}_{4}-\mathrm{CT}-\mathrm{L}_{4}$ & $0.24 \pm 1.7(8)$ & $71.1 \pm 8.1(8)^{\star}$ & 2.6 \\
\hline
\end{tabular}

CaM, but maximal activation was the same (Table 1). On the other hand, M124L-CaM had a similar $K_{\mathrm{D}}$ value to wt-CaM, but activated smMLCK to only $68 \%$ of the activity achieved with wt-CaM (Table 1), in agreement with the results of George et al., [10] who reported $81 \%$ activation of smMLCK by M124LCaM. The $K_{\mathrm{D}}$ and $P_{\max }$ values for activation of CN by M51Land M124L-CaMs were not significantly different from those for wt-CaM (Table 2).

Met-71 and Met-144 are in homologous positions in the fourth helix of each of the two domains of CaM. The $K_{\mathrm{D}}$ and $P_{\max }$ values for activation of smMLCK by M71L- and M144L-CaMs were not significantly different from those for wt-CaM (Table 1). On the other hand, both mutants exhibited decreased maximal activation of $\mathrm{CN}$, but only M71L-CaM had a $K_{\mathrm{D}}$ value significantly greater than wt-CaM (Table 2).

Met-72 and Met-145 are also located in homologous positions in the fourth helix of each of the two domains of CaM. In this case, M72L- and M145L-CaMs behaved quite differently in activation of smMLCK. The $K_{\mathrm{D}}$ value for M72L-CaM was approx. 2-fold lower than for wt-CaM and maximal activation by this mutant was slightly (approx. 10\%) lower than that achieved with wt-CaM (Table 1 ). In contrast, the $K_{\mathrm{D}}$ value for M145L-CaM was comparable to that of wt-CaM and the maximal activation was actually greater (approx. $25 \%$ ) than that induced by wt-CaM (Table 1). The parameters for activation of $\mathrm{CN}$ by these two mutants, however, were indistinguishable from each other and from wt-CaM (Table 2).

Finally, the activation properties of a mutant $\mathrm{CaM}$ in which all four Met in the C-terminal domain were replaced by Leu were investigated. The $K_{\mathrm{D}}$ values for smMLCK and $\mathrm{CN}$ activation by this $\mathrm{M}_{4}-\mathrm{CT}-\mathrm{L}_{4}-\mathrm{CaM}$ quadruple mutant were indistinguishable from the respective wt-CaM values. Maximal activation of both target enzymes by this mutant was significantly (30-40\%) less than maximal activation by wt-CaM (Tables 1 and 2).

\section{DISCUSSION}

Although Meador et al. [14] found that Met-76 is $<4 \AA$ from an Arg in the smMLCK peptide, the properties of M76L-CaM support the earlier conclusion that Met-76, located in the central linker connecting the two domains of $\mathrm{CaM}$, does not play an important role in binding to or activation of target enzymes. Thus M76Q-CaM activated CaM-kinase II, CaM kinase IV and smMLCK [9], and M76L-CaM activated cyclic nucleotide phosphodiesterase (PDE) [8], smMLCK and CN (this study) just 
like wt-CaM. Furthermore, the solution structure of $\mathrm{CaM}$ in complex with CaM-binding peptide derived from skMLCK, and the ${ }^{13} \mathrm{C}$ and ${ }^{1} \mathrm{H}$ NMR chemical shifts of CaM Met methyl groups upon binding either the skMLCK, PDE or cNOS peptide, indicate that Met-76 does not interact with the peptide [5,8,28,29].

Chin and Means [9] examined Met $\rightarrow$ Gln mutants which change both the shape and hydrophobicity of this residue and found that large decreases in the affinity for and activation of smMLCK, CaM kinase II and CaM kinase IV occur most often for mutations in the C-terminal domain of CaM. The M124Q mutant and, to a lesser extent, the M109Q mutant gave the largest and most consistent decreases in their work. These results led them to propose a crucial role for the hydrophobic interaction between Met-124 and the N-terminal hydrophobic anchor residue in the target peptide. Our results for M124L$\mathrm{CaM}$ are consistent with this proposal since this mutant, in which the hydrophobicity of this amino acid is not reduced, has the same affinity as wt-CaM for nNOS [10], smMLCK, CN (this study) and PDE [8]. However, the M124L mutant reduces the maximal activation of nNOS [10] and smMLCK but not of $\mathrm{CN}$ or PDE. With smMLCK, the C-terminal quadruple mutant $\left(\mathrm{M}_{4}\right.$ $\mathrm{CT}-\mathrm{L}_{4}-\mathrm{CaM}$ ) also confirms the importance of Met-124 since it acts like M124L, significantly reducing the maximal activation without a significant decrease in affinity. However, $\mathrm{M}_{4}-\mathrm{CT}-\mathrm{L}_{4}$ $\mathrm{CaM}$ decreased the maximal activation of $\mathrm{CN}$ even though M124L-CaM does not decrease the maximal activation of this enzyme. Instead, the decrease in $\mathrm{CN}$ maximal activation observed for $\mathrm{M}_{4}-\mathrm{CT}-\mathrm{L}_{4}-\mathrm{CaM}$ reflected the decreases observed for $\mathrm{M} 109 \mathrm{~L}$ and M144L. The Met $\rightarrow$ Leu mutation changes the shape of this amino acid as much as the Met $\rightarrow$ Gln mutation which, in the case of smMLCK for Met-124, but not CN, must perturb the conformation sufficiently to decrease the activation. More Nthan C-terminal Met $\rightarrow$ Leu mutations affect the activation parameters for smMLCK, CN (this study) and PDE [8], implying that the importance of interactions between specific Met in CaM and the target enzyme is dependent on the particular target enzyme. Also, an N-terminal mutant, M36L-CaM, gave the largest decrease in affinity for PDE to which it bound with such low affinity (more than 10-fold weaker than wt-CaM) that it was not practical to reach high enough concentrations of the M36L$\mathrm{CaM}$ to measure the maximal percentage activation with this mutant [8].

Since proteolytic removal of the autoinhibitory domain of several CaM target enzymes such as $\mathrm{CN}$ or smMLCK generates constitutively active fragments, simple models of CaM-induced activation are based on displacement of the autoinhibitory domain from the active site by binding of CaM. In this case, all of the mutants would be expected to activate the target enzyme maximally (since they all bind) when added at concentrations sufficient to saturate the enzyme. This is clearly not the case since several mutants failed to activate to the same extent as wt-CaM, even though some of them bound as tightly to the target enzyme as did wt-CaM. Furthermore, the $\mathrm{M}_{4}-\mathrm{CT}-\mathrm{L}_{4}$ mutant bound to smMLCK and $\mathrm{CN}$ as tightly as CaM, consistent with the properties of the corresponding point mutants, but this quadruple mutant maximally activated smMLCK and $\mathrm{CN}$ to significantly lower levels than wt-CaM. These results indicate that there must also be a subtle change in the active site induced by CaM binding and this is different for some of the mutants than for wt-CaM. This conclusion is substantiated by the interesting property of the M145L-CaM mutant which actually activates smMLCK more than does wt-CaM.

From this comparative study of the activation of two CaM target enzymes by a variety of CaM mutants, small but significant differences between the activation parameters for wt-CaM and several of the Met $\rightarrow$ Leu mutants were observed and three patterns emerged: (1) maximal activation of smMLCK by M145L-CaM was significantly greater than maximal activation by wt-CaM (Figure 1; Table 1); (2) comparison of the data in Tables 1 and 2 indicates that several mutations which modify the activation of one target enzyme do not affect the other; and (3) mutations in homologous positions of the two CaM domains show similar effects on the binding and activation parameters for activation of $\mathrm{CN}$ but, in a few cases, different effects on the binding and activation parameters for activation of smMLCK. Again, this emphasizes the differences in the interactions between $\mathrm{CaM}$ and different target enzymes.

This work was supported by grants from the Medical Research Council of Canada (MT-13101 to M.P.W. and MT-9673 to H.J.V.). M.P.W. and H.J.V. are Medical Scientists of the Alberta Heritage Foundation for Medical Research.

\section{REFERENCES}

1 Walsh, M. P. (1994) Mol. Cell. Biochem. 135, 21-41

2 Crivici, A. and Ikura, M. (1995) Annu. Rev. Biophys. Biomol. Struct. 24, 85-116

3 Vogel, H. J. and Zhang, M. (1995) Mol. Cell. Biochem. 149/150, 3-15

4 Babu, Y. S., Bugg, C. E. and Cook, W. J. (1988) J. Mol. Biol. 204, 191-204

5 Ikura, M., Clore, G. M., Gronenborn, A. M., Zhu, G., Klee, C. B. and Bax, A. (1992) Science 256, 632-638

6 O'Neil, K. T. and DeGrado, W. F. (1990) Trends Biochem. Sci. 15, 59-64

7 Gellman, S. H. (1991) Biochemistry 30,6633-6635

8 Zhang, M., Li, M., Wang, J. H. and Vogel, H. J. (1994) J. Biol. Chem. 269, 15546-15552

9 Chin, D. and Means, A. R. (1996) J. Biol. Chem. 271, 30465-30471

10 George, S. E., Su, Z., Fan, D., Wang, S. and Johnson, J. D. (1996) Biochemistry 35, 8307-8313

11 Chin, D., Sloan, D. J., Quiocho, F. A. and Means, A. R. (1997) J. Biol. Chem. 272, $5510-5513$

12 Fauchere, J.-L. and Pliska, V. (1983) Eur. J. Med. Chem. - Chim. Ther. 18, 369-375

13 Sharp, K. A., Nicholls, A., Friedman, R. and Honig, B. (1991) Biochemistry 30, 9686-9697

14 Meador, W. E., Means, A. R. and Quiocho, F. A. (1992) Science 257, 1251-1255

15 Watterson, D. M., Harrelsone, Jr., W. B., Keller, P. M., Sharief, F. and Vanaman, T. C. (1976) J. Biol. Chem. 251, 4501-4513

16 Litwin, C. M. E., Cheng, H. and Wang, J. H. (1991) J. Biol. Chem. 266, 2557-2566

17 Yokoyama, N. and Wang, J. H. (1991) J. Biol. Chem. 266, 14822-14829

18 Klee, C. B., Krinks, M. H., Manalan, A. S., Cohen, P. and Stewart, A. A. (1983) Methods Enzymol. 102, 227-244

19 Hubbard, M. J. and Klee, C. B. (1989) Biochemistry 28, 1868-1874

20 Newton, D. L., Oldewurtel, M. D., Krinks, M. H., Shiloach, J. and Klee, C. B. (1984) J. Biol. Chem. 259, 4419-4426

21 Ngai, P. K., Carruthers, C. A. and Walsh, M. P. (1984) Biochem. J. 218, 863-870

22 Rosner, B. (1995) Fundamentals of Biostatistics, Duxbury Press, Toronto

23 Adelstein, R. S. and Klee, C. B. (1981) J. Biol. Chem. 236, 7501-7509

24 Malencik, D. A., Anderson, S. R., Bohnet, J. L. and Shalitin, Y. (1982) Biochemistry 21, 4031-4039

25 Walsh, M. P., Hinkins, S., Flink, I. L. and Hartshorne, D. J. (1982) Biochemistry 21, 6890-6896

26 Malencik, D. A. and Anderson, S. R. (1986) Biochemistry 25, 709-721

27 Hubbard, M. J. and Klee, C. B. (1987) J. Biol. Chem. 262, 15062-15070

28 Siivari, K., Zhang, M., Palmer III, A. G. and Vogel, H. J. (1995) FEBS Lett. 366, 104-108

29 Zhang, M., Yuan, T., Aramini, J. and Vogel, H. J. (1995) J. Biol. Chem. 270 , 20901-20907 